\title{
Leadership Challenges and Collaborative Opportunities Unveiled Through COVID-19
}

\author{
Gayle Brazeau, PhD'
}

ABSTRACT

It is often said that challenges can be viewed as opportunities in disguise.

This is certainly true as we, the Colleges and Schools of Marshall University, Marshall Health, Cabell Huntington Hospital and Mountain Health Network, independently and collectively navigate the uncharted waters associated with COVID-19.
Author affiliations are listed at the end of this article.

Correspondence to: Gayle Brazeau, PhD Marshall University School of Pharmacy brazeau@marshall.edu

\section{KEYWORDS}

COVID-19, Physician-pharmacist collaborative management (PPCM)

It is often said that challenges can be viewed as opportunities in disguise. This is certainly true as we, the Colleges and Schools of Marshall University, Marshall Health, Cabell Huntington Hospital and Mountain Health Network, independently and collectively navigate the uncharted waters associated with COVID-19. The daily commitment and passion of our clinicians, scientists and educators is creatively focused on maintaining the health and wellness of our patients, students and colleagues. Yet, these times have also unveiled future challenges that we in West Virginia, the Tri-State area, and beyond will need to resolve in order to minimize disruption to our daily lives. Our future will be based in large part upon how well we can effectively work as interprofessional teams of clinicians, educators and scientists. Several challenges in providing timely and appropriate healthcare have been revealed with COVID-19. These and other currently unforeseen challenges will be the ones we will need to tackle in order to maintain successful patient outcomes in our urban and rural communities.

It is clear we will need to continue to provide health care professionals that will serve our urban and rural communities in Appalachia and beyond. The health professional schools of Marshall University remain committed to this endeavor. We work to attract and support students in their education as future physicians, pharmacists, physician assistants, nurses, physical therapists, medical laboratory technicians, social workers, public health workers, dieticians, audiologists and speech-language pathologists. While we cannot clearly determine the totality of the economic impact of COVID-19 on our students' abilities to pursue or complete their studies at this time, our current clinicians, faculty members, and friends are working to minimize disruptions. We have learned in the current time the importance of enhancing and providing stable internet connections for all our students across West Virginia and beyond. Additionally, this is a time for the Marshall University health science programs to collectively work with our communities to encourage even more students to consider professions in healthcare particularly as nurses, pharmacists and other health care first responders. An advantage of Marshall University is the number of undergraduate and graduate health professions programs that students can pursue and the great potential for interprofessional education and practice in our care of patients. 
A second area of concern that has been exacerbated and most likely will continue in the future is key drug shortages in our hospitals and community pharmacies. Drug shortages have always been a part of contemporary healthcare settings in hospitals and community pharmacies. The reason for drug shortages can include off-shore manufacturing and quality problems resulting in shortages in active pharmaceutical ingredients, delays, and discontinuations. For example, COVID-19 has resulted in disruptions of at least 156 critical drugs manufactured in China, Italy, India or other countries as reported by the Center for Infectious Disease Research and Policy (CIDRAP) at the University of Minnesota. ${ }^{1}$ The Food and Drug Administration (FDA) is responsible for working closely with the manufacturers to limit or reduce the impact of these shortages. However, with COVID-19 we are seeing additional disruptions in the supply chain of essential drugs and testing reagents that may continue well beyond the current situation. ${ }^{2}$ Hospital pharmacists and pharmacies are well acquainted with the challenges of drug shortages particularly intravenous fluids and injectable drugs. Hospital pharmacies often have dedicated staff (pharmacists and associated staff) to serve as a resource for the clinicians while managing and minimizing these shortages in patient care activities in their institution or healthcare system. These drug shortages are listed in two real-time on-line references; 1 ) the American Society of HealthSystem Pharmacists Drug Shortages ${ }^{3}$ and 2) the Food and Drug Administration Drug Shortages ${ }^{2}$.

However, in the ambulatory care and community pharmacy settings, a system to minimize the effect of drug shortages on patient care is not as well organized in the current health care system. The challenge arises when the clinician writes the prescription and the patient goes to the community pharmacy only to find out that this specific drug is not available due to the shortage. The next step is the pharmacist taking time to call community pharmacies in the area to see if they have the drug and/or to call the physician's office to discuss the shortage situation and possible therapeutic options. This comes at a cost, however, as time is lost for the pharmacist, physician and staff members of the clinic and pharmacy that would otherwise be used for direct patient care activities. Imagine a situation where one's home is in a rural setting, one can certainly envision how a drug shortage of specific orally administrated drugs could impact on the treatment of chronic disease conditions, particularly if there is a limited number of pharmacies in their community.

With an eye towards solving this challenge now and in the future, this is an excellent time for the Marshall University School of Pharmacy and the Joan C. Edwards School of Medicine to collaborate with community pharmacies and pharmacists in developing strategies to identify and minimize these drug shortages. Physicians and pharmacists in ambulatory care clinics working with pharmacists in their community pharmacies are excellent partners who can provide insight into developing strategies that can be used to solve this challenge. This solution could involve the development of an online searchable resource that would provide current upto-date drug shortages of relevant drugs used in the clinic setting combined with the availability of these specific drugs in community pharmacies. This would involve two key components, 1) pharmacists and physicians working together to identify therapeutic options for specific drug shortages that could be used by their colleagues if a drug is not available and 2) a tool that could be used by clinic physicians and nurses as well as community pharmacists to provide the names of community pharmacies where the patients could find the prescribed drug. As a starting point, the School of Pharmacy and the School of Medicine are working together to develop the on-line resource for therapeutic options for drug shortages that could be used by clinicians and nurses in the clinics and by community pharmacists. We are also reaching out to community pharmacies to establish efficient and real-time approaches to collect information on the availability of drugs that may be in short supply in our area and eventually throughout the state.

A third area of concern that we may see as a result of COVID-19 is the continued closure of pharmacies in rural and urban communities resulting in these areas being considered a pharmacy desert. A pharmacy desert is defined as any area where patients have poor access to prescription medications. This lack of access can be a result of long distances to get to the pharmacy because of closures, limited 
transportation, insurance status, and lower incomes. ${ }^{4}$ It has been suggested that nearly 100 million Americans lack adequate access to pharmacies. ${ }^{5}$ West Virginia is no exception in that we have seen a continued decline in the number of pharmacies in both our urban and rural communities combined with long distances between our communities. One reason that continues to lead to the loss of community pharmacies around the country is the declining reimbursements that a pharmacy receives when filling the prescription order; this is having a significant impact on the business' financial stability.

The impact of COVID-19 on future pharmacy closures is not known at this stage. Yet there is concern that many of these struggling pharmacies have become frontline sources of patient care and information in their communities. What will happen in the future if there are shortages in available prescribed drugs as well as in new drugs and vaccinations? Similar declines have also been observed in access to patient care in rural and underserved settings. We continue to see few physicians choosing to work in these areas combined with the closure of community hospitals and health care facilities in West Virginia and the nation..$^{6-8}$ Like on community pharmacies, the impact of COVID-19 on rural patient care is also unknown at this stage, but this will provide yet another strain on already a fragile health care system in rural and underserved areas. The decline of primary care and pharmacy services combined with the impact of COVID-19 in our rural and underserved communities provides another leadership opportunity for our Schools of Pharmacy and Medicine and for individual physicians and pharmacists to work together. Physician-pharmacist collaborative management (PPCM) has been shown to improve the management of patients with chronic diseases. ${ }^{9}$ This is particularly relevant to ambulatory care clinics as recently approved West Virginia legislation allows pharmacists to bill for services provided that are within their scope of practice if these benefits would be provided for such services performed by other health care providers. ${ }^{10}$

COVID-19 has certainly tested our educational and health care systems. Yet, we should also consider how this situation has provided us with openings to now advance innovative practice tools and sites in West Virginia. We, pharmacists and physicians, collectively need to work with our local governments, the West Virginia Legislature and the Governor's Office to ensure the availability of patient care provided at local clinics and pharmacies. While COVID-19 has and continues to be a challenge to providing successful patient outcomes, this can also be the time for physicians and pharmacists to think creatively about new strategies for enhanced collaboration focused always on optimizing patient care in West Virginia and beyond.

\section{AUTHOR AFFILIATIONS}

1. Marshall University School of Pharmacy, Huntington, West Virginia

\section{REFERENCES}

1. Turner T, Miller E. COVID-19 and Drug Shortages: How to Prepare. Drugwatch, Available from https://www.drugwatch.com/news/2020/04/06/ covid-19-drug-shortages-how-to-prepare/. April 6, 2020. Accessed April 22, 2020.

2. Drug Shortages Food and Drug Administration [Internet] Washington. [Cited 2020, April 1] Available at https://www.fda.gov/drugs/drugsafety-and-availability/drug-shortages. Accessed April 22, 2020.

3. Drug Shortages American Society of Healthsystem Pharmacists [Internet] Bethesda [Cited 2020, April 20], Available at https://www.ashp. org/Drug-Shortages. Accessed April 22, 2020.

4. Gebhart F. What is a pharmacy desert? Drug Topics [Internet] 2019 Sept [cited 2019 Sept 24; 163(9). Available at https://www.drugtopics.com/ chains-business/what-pharmacy-desert.

5. Gebhart F. The growing problem of pharmacy deserts. Drug Topics [Internet] 2019 Sept [cited 2019 Sept 24; 163(9). Available at https://www. drugtopics.com/latest/growing-problempharmacy-deserts.

6. West Virginia Rural Health Association. National Center for the Analysis of Healthcare Data. Health care in West Virginia. A Workforce Supply and Demand Analysis 2018 August. Available at https://wvrha.org/wp-content/ uploads/2019/03/2018-FINAL-WV-Workforce- 
updated-9_26.pdf.

7. Frakt $A B$, The rural hospital problem. JAMA. 2019;321(23):2271-2.

8. West Virginia Health and Human Resources State Office of Rural Health. Creating a culture of health in rural West Virginia: State rural health plan 2018-2022. 2018 Jan Available at https:// wvrha.org/wp-content/uploads/2017/08/2018State-Rural-Health-Plan-Final.pdf.

9. Hwang AY, Gums TH, Gums JG. The benefits of physician-pharmacist collaboration. J Fam Pract. 2017;66 (12):E1-E8.

10. Providing Benefits to Pharmacists for Rendered Care, §33 - 53 - 1 West Virginia Legislature (June $5,2020)$. 